\title{
Algunos digéneos de Rhinella marina (Anura: Bufonidae) en Colombia
}

\author{
Some digeneans of Rhinella marina (Anura: Bufonidae) in Colombia
}

\author{
Mauricio Bechara ${ }^{1}$ e Imelda Vélez ${ }^{2 *}$ \\ ${ }^{1}$ Universidad Tecnológica del Chocó,Carrera 22 Núm. 18 B-10-B/Nicolás Medrano A.A. 292, Quibdó-Chocó-Colombia. \\ ${ }^{2}$ Programa de Estudio y Control de Enfermedades Tropicales (PECET). Torre 2, Laboratorio 730. Sede de Investigación Universitaria (SIU), \\ Universidad de Antioquia. Calle 62 Núm. 52-69. Medellín, Colombia. \\ *Correspondencia: ivelez@matematicas.udea.edu.co
}

Resumen. Se estudiaron 40 sapos Rhinella marina Linnaeus, 1758 (24 machos y 16 hembras) del Valle de Aburrá, Antioquia, Colombia, 8 de los cuales se encontraron parasitados por 2 especies de digéneos: Pseudosonsinotrema chabaudi (Caballero y Caballero, 1969) Sullivan, 1974 (Pleurogenidae) (primer registro para Colombia y para el hospedero) y Mesocoelium monas (Rudolphi, 1819) Freitas, 1958 (Brachycoeliidae), previamente registrado para este país. De las 2 especies de helmintos recolectadas, la que alcanzó los mayores niveles de prevalencia (40\%) y abundancia media (14.7) fue P. chabaudi en San Antonio de Prado, y la intensidad promedio más elevada fue para M. monas, en los sapos de Barbosa (46.7).

Palabras clave: Digenea, Pseudosonsinotrema chabaudi, Mesocoelium monas, Neotropico.

\begin{abstract}
Eight of 40 Rhinella marina Linnaeus, 1758 (24 males and 16 females) from Aburrá Valley, Antioquia, Colombia, were infected with 2 digenea species: Pseudosonsinotrema chabaudi (Caballero y Caballero, 1969) Sullivan, 1974 (Pleurogenidae), which represents new host and country records, and Mesocoelium monas (Rudolphi, 1819) Freitas, 1958 (Brachycoeliidae), which was previously reported for the country. Pseudosonsinotrema chabaudi in San Antonio de Prado reached the highest prevalence levels (40\%) and mean abundance (14.7), whereas the highest mean intensity was recorded for M. monas in cane toads from Barbosa (46.7).
\end{abstract}

Key words: Digenea, Pseudosonsinotrema chabaudi, Mesocoelium monas, Neotropic.

\section{Introducción}

Pocos estudios se conocen sobre digéneos en anuros colombianos. Hasta la fecha se han registrado 5 especies: Haematoloechus medioplexus Stafford 1902 en Rana palmipes Spix, 1824 de Cali (Uribe-Piedrahíta, 1948); Glypthelmins robustus Brooks, 1976; Creptotrema lynchi Brooks 1976 y Gorgoderina diaster Lutz, 1926 en Rhinella marina Linnaeus, 1758 (=Chaunus marinus= Bufo marinus), colectados en los departamentos de Atlántico y Huila (Brooks, 1976), y Mesocoelim monas Rudolphi, 1819 en R. marina del departamento del Chocó (Bechara-Escudero et al., 2007).

La fauna helmintológica de $R$. marina ha sido ampliamente estudiada; hasta la fecha se han realizado numerosos registros a lo largo de su distribución, tanto nativa como introducida. Esta información, compilada en 3 ocasiones (Speare, 1990; Barton, 1997; Espinoza-Jiménez et al., 2007), establece en 113 el número de especies de helmintos que parasitan este anfibio en todo el mundo, de las cuales 34 pertenecen al grupo de los digéneos.

El objetivo del presente estudio fue identificar los

Recibido: 27 noviembre 2008; aceptado: 21 octubre 2009 digéneos parásitos de $R$. marina en el Valle de Aburrá, departamento de Antioquia, Colombia y determinar su prevalencia, intensidad media y abundancia.

\section{Materiales y métodos}

Entre 2003 y 2005 se colectaron 40 sapos $R$. marina en 4 estaciones del Valle de Aburrá, Departamento de Antioquia, Colombia: Barbosa $\left(6^{\circ} 27^{\prime} \mathrm{N} ; 7^{\circ} 17^{\prime} \mathrm{O}, 1400\right.$ $\mathrm{m}$ snm); San Antonio de Prado (6 $6^{\circ} 10^{\prime} \mathrm{N} ; 7^{\circ} 30^{\prime} \mathrm{O}, 2090 \mathrm{~m}$ snm); Bello $\left(6^{\circ} 19^{\prime} \mathrm{N} ; 7^{\circ} 32^{\prime} \mathrm{O}, 1425 \mathrm{~m}\right.$ snm) y Sabaneta $\left(06^{\circ} 09^{\prime} \mathrm{N} ; 75^{\circ} 36^{\prime} \mathrm{O}, 1585 \mathrm{~m} \mathrm{snm}\right)$. Los hospederos fueron medidos (LRC: Longitud rostro cloaca en $\mathrm{cm}$ ), sexados y sacrificados en el laboratorio. El tubo digestivo fue aislado durante 1 hora en solución fisiológica al $0.6 \%$ para permitir que los parásitos se desprendieran de las paredes intestinales (Werding, com. pers.); posteriormente, el tubo se abrió en forma longitudinal y se examinó.

Los parásitos fueron fijados en FAA, se tiñeron con carmín borácico y carmín de Meyer, se aclararon en xilol, Berlese y/o salicilato de metilo, después se montaron en placas permanentes con bálsamo de Canadá y Entellan. Los digéneos se ilustraron bajo cámara lúcida; las medidas 
están dadas en mm (excepto los huevos, en $\mu \mathrm{m}$ ). Los ejemplares de referencia se depositaron en la Colección de Tremátodos Digéneos de la Universidad de Antioquia (CTUA116), Laboratorio de Malacología Médica y Tremátodos del Programa de Estudio y Control de Enfermedades Tropicales (PECET), Medellín, Colombia. Los parámetros ecológicos utilizados para cada especie de digéneo y estación de muestreo se calcularon de acuerdo con Bush et al. (1997).

\section{Resultados}

En cada estación de muestreo se colectaron 10 hospederos, 40 individuos en total (24 machos y 16 hembras, $\mathrm{LCR}=9.8-15.8 \mathrm{~cm}$; peso: 79-279 g). Se encontraron 8 anuros parasitados (20\%) en 3 de las 4 estaciones de muestreo evaluadas; los digéneos intestinales hallados fueron Pseudosonsinotrema chabaudi (Caballero y Caballero, 1969) Sullivan, 1974 (Fig. 1), en San Antonio de Prado y Mesocoelium monas Rudolphi, 1819, en Barbosa y Bello; en Sabaneta no se encontraron hospederos parasitados. En el Cuadro 1 se comparan las medidas de P. chabaudi con las de ejemplares colectados en Costa Rica (Caballero y Caballero, 1969; Sullivan, 1971). Los parámetros que caracterizaron las infecciones encontradas en los hospederos de cada estación de muestreo se presentan en el Cuadro 2. De las 2 especies de helmintos recolectadas, $P$. chabaudi fue la que alcanzó los mayores niveles de prevalencia y abundancia media, mientras que la intensidad promedio más elevada fue registrada para $M$. monas en los sapos de Barbosa.

\section{Discusión}

Pseudosonsinotrema chabaudi se identificó con base en las características morfológicas definidas para esta especie por Caballero y Caballero (1969), quienes la describieron como Brenesia chabaudi Caballero y Caballero, 1969. Posteriormente, el género Brenesia fue sinonimizado con Pseudosonsinotrema por Sullivan (1974), transfiriendo la especie de Caballero y Caballero a este último género. La validez de la especie está reconocida en la actualidad (Rodríguez-Ortíz et al., 2004; Lotz y Font, 2008), caracterizándose por su vesícula excretora con forma de $\mathrm{V}$ a Y, a la cual se ajustan los ejemplares del presente estudio y cuyas ramas son anchas, como se observa en la figura 1. Mesocoelium monas se determinó de acuerdo con la redescripción de la especie que realizaron Travassos et al. (1969).

Pseudosonsinotrema chabaudi en Colombia alcanzó

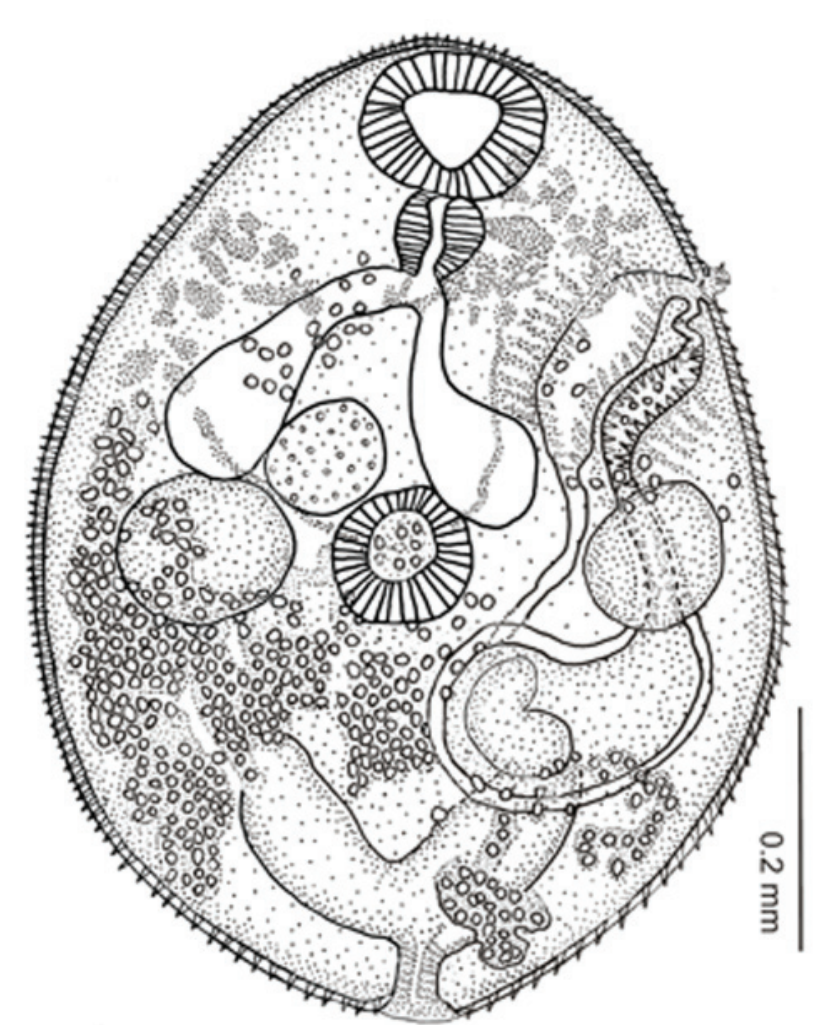

Figura 1. Pseudosonsinotrema chabaudi (Digenea: Pleurogenidae). Vista ventral en $R$. marina del Valle de Aburrá, Departamento de Antioquia. Colombia.

niveles de infección más altos que los registrados para Lithobates pipiens Schreber, 1782 (=Rana pipiens Schreber, 1782) en Costa Rica por Sullivan (1971), quien encontró 22 individuos de $P$. chabaudi en 4 de 16 ranas examinadas $(25 \%$; 1.37 digéneos por hospedero revisado y 5.5 gusanos por hospedero parasitado). Los valores de prevalencia de M. monas en Bello (10\%) y Barbosa (30\%) son similares a los registrados para $R$. marina en el Chocó, variando entre 10\%-40\% (Bechara y Asprilla, 2007). Ambas circunstancias podrían indicar preferencia de $R$. marina por presas y hábitats acuáticos, como lo sugieren Espínola-Novelo y Guillén-Hernández (2008). En nuestros muestreos no se encontraron anuros parasitados simultáneamente con M. monas y P. chabaudi.

Pseudosonsinotrema chabaudi es registro nuevo para $R$. marina; con ello se amplía a 114 el número de especies descritas para este hospedero a lo largo de su distribución natural e introducida (véase Espinoza-Jiménez et al., 2007); asimismo, su hallazgo en Colombia amplía su distribución geográfica en la región neotropical, pues previamente sólo se había colectado en Costa Rica (Caballero y Caballero, 1969). 
Cuadro 1. Comparación morfométrica de Pseudosonsinotrema chabaudi de Costa Rica y Colombia

\begin{tabular}{|c|c|c|c|}
\hline \multirow[b]{2}{*}{ Autor } & \multicolumn{2}{|c|}{ Costa Rica } & \multirow{2}{*}{$\begin{array}{c}\text { Colombia } \\
\text { Pseudosonsinotrema chabaudi } \\
\text { Presente estudio } \\
\mathrm{n}=10\end{array}$} \\
\hline & $\begin{array}{c}\sin . \text { Brenesia chabaudi } \\
\text { Caballero y Caballero (1969) } \\
n=?\end{array}$ & $\begin{array}{l}\text { sin. Pseudosonsinotrema } \\
\text { echinophallus } \\
\text { Sullivan (1971) } \mathrm{n}=?\end{array}$ & \\
\hline Hospedero & Litobathes pipiens & L. pipiens & Rhinella marina \\
\hline Localidad & $\begin{array}{l}\text { Provincia de Heredia } \\
\text { (Puerto Viejo, Sarapiqui) }\end{array}$ & $\begin{array}{l}\text { Provincia de Cartago } \\
\text { (Turrialba) }\end{array}$ & $\begin{array}{l}\text { Departamento de Antioquia } \\
\text { (San Antonio de Prado) }\end{array}$ \\
\hline $\begin{array}{l}\text { Cuerpo } \\
\text { Espinas (Tegumento) }\end{array}$ & $\begin{array}{c}0.714-0.743 \times 0.400-0.500 \\
0.007 \times 0.002\end{array}$ & $\begin{array}{c}0.64-0.84(0.72) \times 0.44-0.57 \\
(0.51)\end{array}$ & $\begin{array}{c}0.737-0.871(0.787 \pm 0.041) \times \\
0.510-0.627(0.569 \pm 0.046)\end{array}$ \\
\hline Ventosa oral & $0.109-0.126 \times 0.116-0.116$ & $\begin{array}{c}0.088-0.11(0.1) \times 0.095-0.13 \\
(0.11)\end{array}$ & $\begin{array}{c}0.078-0.126(0.099 \pm 0.023) \times \\
0.165-0.141(0.137 \pm 0.019)\end{array}$ \\
\hline Acetábulo & $0.119-0.139 \times 0.126-0.129$ & $\begin{array}{c}0.11-0.13(0.12) \times 0.11-0.13 \\
(0.12)\end{array}$ & $\begin{array}{c}0.110-0.157(0.134 \pm 0.015) \times \\
0.118-0.141(0.129 \pm 0.012)\end{array}$ \\
\hline V. oral/Acetábulo & $1: 1.09$ x 1:1.08-1:1.1 x $1: 1.1$ & $1.0: 1.02$ & \\
\hline Boca & $0.068-0.071 \times 0.078-0.082$ & & \\
\hline Faringe & $0.034-0.041 \times 0.037-0.037$ & $0.032-0.041(0.36) \times 0.03-0.037$ & $0.039-0.078(0.059 \pm 0.012) \times$ \\
\hline Esófago & $0.044-0.068 \times 0.014-0.017$ & & 0.047-0.071 (0.059 \pm 0.008$)$ \\
\hline Poro genital & D $0.014-0.014$ & & \\
\hline Testículo derecho & $0.105-0.146 \times 0.075-0.095$ & $\begin{array}{c}0.094-0.17(0.13) \times 0.076-0.14 \\
(0.11)\end{array}$ & $\begin{array}{c}0.118-0.196(0.146 \pm 0.033) \times \\
0.094-0.149(0.115 \pm 0.028)\end{array}$ \\
\hline Testículo izquierdo & $0.085-0.109 \times 0.051-0.088$ & $\begin{array}{c}0.11-0.16(0.14) \times 0.068-0.13 \\
(0.094)\end{array}$ & $\begin{array}{c}0.094-0.157(0.129 \pm 0.022) \times \\
0.078-0.141(0.105 \pm 0.026)\end{array}$ \\
\hline \multirow[t]{4}{*}{ Saco del cirro } & Parte posterior & Porción basal bulbosa & \\
\hline & $0.109-0.0119 \times 0.146-0.167$ & $\begin{array}{c}0.35-0.52(0.43) \times 0.083-0.11 \\
(0.097)\end{array}$ & $\begin{array}{c}0.188-0.235(0.226 \pm 0.021) \times \\
0.118-0.157(0.136 \pm 0.020)\end{array}$ \\
\hline & $\begin{array}{c}\text { Parte anterior } \\
\text { (pars prostática?) }\end{array}$ & & \\
\hline & $0.214-0238 \times 0.024-0.037$ & & \\
\hline Vesícula seminal & $0.075-0.112 \times 0.075-0.112$ & & \\
\hline Cirro espinoso & $0.014 \times 0.017$ & & \\
\hline Ovario & $0.099-0.160 \times 0.051-0.088$ & $\begin{array}{c}0.098-0.13(0.12) \times 0.051-0.12 \\
(0.092)\end{array}$ & $\begin{array}{c}0.078-0.141(0.107 \pm 0.022) \mathrm{x} \\
0.078-0.157(0.115 \pm 0.027)\end{array}$ \\
\hline Huevos & $\begin{array}{c}0.019-0.019 \times 0.008-0.009 \\
\mu \mathrm{m}\end{array}$ & $19-22(20) \times 8-10(9) \mu \mathrm{m}$ & $\begin{array}{l}17.9-25.9(23.1 \pm 2.0) x \\
10-15.9(13.4 \pm 1.9) \mu \mathrm{m}\end{array}$ \\
\hline Metratermo & $0.214-0.309 \times 0.075-0.078$ & & $\begin{array}{c}0.196-0.314(0.245 \pm 0.059) \times \\
0.039-0.094(0.071 \pm 0.028)\end{array}$ \\
\hline Vesícula excretora & forma de $\mathrm{V}$ & forma de $\mathrm{Y}$ & forma de V a Y \\
\hline
\end{tabular}

D: diámetro; largo x ancho; mínimo-máximo en mm (promedio \pm desviación estándar)

\section{Agradecimientos}

A Luis García-Prieto de la Colección Nacional de Helmintos del Instituto de Biología, UNAM, a la Universidad de Antioquia por su apoyo. A Carolina Lenis del Laboratorio de Malacología y Tremátodos (PECET, Universidad de Antioquia), por sus sugerencias al texto.

\section{Literatura citada}

Barton, D. 1997. Introduced animals and they parasites. The cane toad, Bufo marinus in Australia. Australian Journal of Ecology 22:316-324.

Bechara-Escudero, M. y S. Asprilla-Murillo. 2007. Tremátodos Digéneos de Chaunus marinus (Anura:Bufonidae) en 
Cuadro 2. Caracterización de los digeneos de R. marina del Valle de Aburrá, Antioquia, Colombia

\begin{tabular}{lccccc}
\hline \multicolumn{1}{c}{ Digeneos (Estaciones) } & $\begin{array}{c}\text { Número de } \\
\text { digeneos }\end{array}$ & $\begin{array}{c}\text { Prevalencia } \\
(\%)\end{array}$ & $\begin{array}{c}\text { Abundancia } \\
\text { media }\end{array}$ & $\begin{array}{c}\text { Intensidad } \\
\text { media (Intervalo) }\end{array}$ & $\begin{array}{c}\text { Número } \\
\text { de catálogo }\end{array}$ \\
\hline $\begin{array}{l}\text { Mesocoelium monas } \\
\text { Barbosa }\end{array}$ & 140 & 30 & 14 & $46.7(4-119)$ & CTUA116.863.872 \\
Bello & 41 & 10 & 3.1 & 31 & CTUA116.873-882 \\
$\begin{array}{l}\text { Pseudosonsinotrema chabaudi } \\
\text { San Antonio de Prado }\end{array}$ & 148 & 40 & 14.7 & $36.8(19-68)$ & \\
\hline
\end{tabular}

el Municipio de Quibdó, Chocó. Revista Institucional Universidad Tecnológica del Chocó: Investigación, Biodiversidad y Desarrollo 26:13-17.

Brooks, D. R. 1976. Five species of platyhelminths from Bufo marinus L. (Anura:Bufonidae) in Colombia with descriptions of Cretotrema lynchi sp. n. (Digenea: Allocreadiidae) and Glypthelmins robustus sp. n. (Digenea: Macroderoididae). Journal of Parasitology 62:429-433.

Brooks, D. R., V. León-Règagnon, D.A. McLannan y D. Zelmer. 2006. Ecological fitting as a determinant of the community structure of platyhelminth parasites of anurans. Ecology 87:76-85.

Bursey C. R. y S. R. Goldberg. 2004. Cosmocerca vrcibradici n. sp. (Ascaridida: Cosmocercidae), Oswaldocruzia vitti n. sp. (Strongylida: Molineoidae) and other helminths from Prionodactylus eigenmanni and Prionodactylus oshaughnessyi (Sauria: Gymnophthalmidae) from Brazil and Ecuador. Journal of Parasitology 90:140-145

Bush, A. O., K. D. Lafferty, J. M. Lotz y A. W. Shostak. 1997. Parasitology meets ecology on its own terms: Margolis et al. revisited. Journal of Parasitology 83:575-583.

Caballero, E. C. y G. Caballero R. 1969. Un trematode nouveau. Parasite de Rana pipiens Schreber, 1872 de la République de Costa Rica (Amérique Centrale). Annales de Parasitologie (Paris) 44:539-546.

Espínola-Novelo J. F. y S. Guillén-Hernández. 2008. Helminth parasites in Chaunus marinus and Cranopis valliceps (Anura: Bufonidae) from Lagunas Yalahau, Yucatán, Mexico. Journal of Parasitology 94:672-674.

Espinoza-Jiménez, A., L. García-Prieto, D. Osorio-Saravia y V. León-Règagnon. 2007. Checklist of Helminth Parasites of the Cane Toad Bufo marinus (Anura:Bufonidae) from Mexico. Journal of Parasitology 93:937-944.

Goldberg, S. R., C. R. Bursey y S. R. Telford, Jr. 2005. Metazoan endoparasites of four species of lizards Gehyra mutilata, Hemidactylus frenatus (Gekkonidae), Mabuya cumingi, Mabuya multifasciata (Scincidae) and one species of snake Ramphotyphlops braminus (Typhlopidae) from the Philippine Islands. Comparative Parasitology 72:88-101.

Lotz, J. M. y W. F. Font. 2008. Family Pleurigenidae Looss, 1989. In Keys to the Trematoda, A. Rodney, D. Bray, I. Gibson y A. Jones. 2008. The Natural History Museum,
London. 848 p.

Manter, H. W. y M. H. Pritchard. 1964. Mission de zoologie médicale au Maniema (Congo, Léopoldville) (P.L.G. Benoit, 1959). 5. Vermes-Trematoda. Annales du Musee Royale del'Afrique Centrale, Zoologie 132:75-101.

Pérez-Ponce de León, G., V. León-Règagnon, L. García-Prieto, U. Razo-Mendivil y A. Sánchez-Álvarez. 2000. Digenean fauna of amphibians from Central Mexico: neartic and neotropical influences. Comparative Parasitology 67:92-106.

Prudhoe, S. y R. A. Bray. 1982. Platyhelminth parasites of the Amphibia. British Museum (Natural History)/Oxford University Press, London. 371 p.

Ragoo, R. M. y I. R. Omah-Maharaj. 2003. Helminths of the cane toad Bufo marinus from Trinidad, West Indies. Caribbean Journal of Science 39:242-245.

Rodríguez-Ortiz, B., L. García-Prieto y G. Pérez-Ponce de León. 2004. Checklist of the helminth parasites of vertebrates in Costa Rica. Revista de Biología Tropical 52:313-354.

Speare R. 1990. A review of diseases of the cane toad, Bufo marinus, with comments on biological control. Australian Wildlife Research 17:387-410.

Sullivan, J. J. 1971. Pseudosonsinotrema echinophallus sp. n. (Digenea: Pleurogenidae), a new Trematode from Rana pipiens Schreber in Costa Rica. Proceedings of the Helminthological Society of Washington 38:34-37.

Sullivan, J. J. 1974. Pseudosonsinotrema chabaudi (Caballero y C. and Caballero R., 1969) comb. n., a senior Synonym of $P$. echinophallus Sullivan, 1971, from Costa Rican frogs. Proceedings of the Helminthological Society of Washington 41:251.

Travassos, L., J. E. T. Freitas y A. Kohn. 1969. Trematodeos do Brasil. Memorias do Instituto Oswaldo Cruz 67:1-886.

Uribe-Piedrahíta, C. 1948. Contribución al estudio de la parasitología en Colombia, II. Caldasia 5:211-219.

Wong, M. S. y D. A. P. Bundy. 1985. Population distribution of Ochoterenella digiticauda (Nematoda: Onchocercidae) and Mesocoelium monas (Digenea: Brachycoeliidae) in naturally infected Bufo marinus (Amphibia: Bufonidae) from Jamaica. Parasitology 90:457-461.

Yamaguti, S. 1936. Studies on the helminth fauna of Japan. Part 14. Amphibian trematodes. Japanese Journal of Zoology 6:551-576. 